\title{
The World Health Organization's QualityRights materials for training, guidance and transformation: preventing coercion but marginalising psychiatry
}

Fiona Hoare and Richard M. Duffy

\section{Summary}

The World Health Organization has developed training material to support its QualityRights Initiative. These documents offer excellent strategies to limit coercion. However, the negative portrayal of psychiatry, the absolute prohibition on involuntary treatment and the apparent acceptance of the criminalisation of individuals with mental illness are causes for concern.

\section{Keywords}

Human rights; coercion; World Health Organization; QualityRights

\section{Copyright and usage}

(c) The Author(s), 2021. Published by Cambridge University Press on behalf of the Royal College of Psychiatrists.
Fiona Hoare (pictured) is a psychiatry trainee currently working in liaison psychiatry and has a degree in business and law. Richard M. Duffy is a consultant in liaison and perinatal psychiatry, and he is currently undertaking a $\mathrm{PhD}$ in mental health law and human rights.

The World Health Organization (WHO) QualityRights Initiative is doing excellent work in shifting modern psychiatry away from coercive practices, towards rights-based, patient-centred care. ${ }^{1}$ However, some of the content of its materials for training, guidance and transformation ${ }^{1}$ may undermine the practice of psychiatry and compromise the rights of individuals with major mental illness.

\section{Background}

The United Nations Convention on the Rights of Persons with Disabilities (CRPD) and WHO, have sought to reduce coercive measures in mental health treatment. However, the Committee on the Rights of Persons with Disabilities' and the QualityRights Initiative have taken this aim a step further and are seeking to end involuntary treatment, altogether. This position is strongly informed by the Committee on the Rights of Persons with Disabilities' interpretation of Article 12 of the CRPD, which refers to equal recognition before the law. The banning of involuntary treatment is at odds with the majority of jurisdictions current legislation and Freeman et $\mathrm{al}^{2}$ have highlighted how this could actually limit the rights of individuals with mental illness. Such a prohibition is strongly divergent from WHO guidance published within the past 15 years, for example the WHO Resource Book on Mental Health, Human Rights and Legislation (which has now been withdrawn).

In 2012, the WHO developed its QualityRights Initiative and published the WHO QualityRights Tool Kit. This Initiative seeks to translate international human right standards, in particular the CRPD, into practice by influencing policy and building the knowledge and skills to implement person-centred and recovery-based approaches. ${ }^{3,4}$ The development of this toolkit has involved examining current practices worldwide and in doing so has highlighted many important areas for reform.

WHO training.

\section{WHO QualityRights training materials}

In 2019, accompanying materials for training, guidance and transformation were published. ${ }^{1}$ These materials have five core training modules: 'Human rights', 'Mental health and human rights', 'Legal capacity and the right to decide', 'Recovery and the right to health' and 'Freedom from coercion, violence and abuse'. In addition, there are three specialised training modules, 'Recovery practices for mental health and well-being,' 'Strategies to end seclusion and restraint' and 'Supported decision making and advanced planning'.

\section{Supported decision-making}

There are many positive elements in the QualityRights Initiative's training material and their objective of realising and protecting human rights in mental healthcare should be widely embraced. The authors drew on a diverse group of global experts to develop strategies to help integrate the CRPD into mental health practices. In particular, the module on 'Legal Capacity and the Right to Decide' provides excellent discussion on supported decision-making. It highlights jurisdictions where good practice is found, for example, in Sweden and Finland where a 'Personal Ombudsperson' and 'Open Dialogue' are employed with good effect. The training materials discuss many complex topics including advance planning and it briefly discusses the possible utilisation of a 'Ulysses Clause'.

\section{Promoting recovery}

There is an important dialogue on the promotion of mental health, in the module on 'Recovery and the right to health'. The need to improve the often-neglected physical health of people with mental illness is highlighted. There are practical suggestions for promoting recovery and the role of individualised plans is discussed. The guidance materials utilise case studies, debates and exercises that help engage users with the concepts of human rights and recovery. There are useful guidelines for the development of peer support groups and advocacy for mental health. 


\section{Reducing coercive practices}

The availability of the QualityRights training online has the potential to reach a wide range of stakeholders. Importantly, there are excellent suggestions for reducing coercive practices. Such strategies include increased staff training, particularly in communication techniques. There is a useful discussion on the effectiveness of supportive environments and comfort rooms. Response teams are proposed as a way of providing specialised and individualised intervention in periods of distress and reducing restraint and seclusion practices.

\section{Limitations}

Unfortunately, there are three significant limitations, and it is important to address these. First, there is a negative portrayal of psychiatry and psychopharmacology. Second, any involuntary practices are characterised as human rights abuses. Third, there is a disturbing acceptance of the criminalisation of individuals with severe mental illness.

\section{Portrayal of psychiatry and psychopharmacology}

Psychiatry is portrayed in an unbalanced manner, with multiple negative descriptions throughout the guidance materials. Psychiatrists are mentioned at least 16 times, 13 of those references are negative. Practitioners are portrayed as unsympathetic, dismissive and heavy handed in prescribing medication. This is stigmatising of the profession and could create a further barrier to individuals accessing healthcare.

Similarly, psychotropic medication is represented in a highly negative light. There are multiple references to the adverse effects of medication, at least 14 references to medications were identified none of which mentioned the advantages of pharmacological interventions. Psychotropics have the potential to dramatically improve the quality of an individual's life. Although they are associated with both risks and benefits, this is true of all medication. Their depiction in the training materials does not reflect their robust evidence base.

Along with a misleading portrayal of psychiatry, the authors represent an idealised depiction of psychosocial interventions. One of many examples is found in the 'Legal Capacity and Right to Decide' module, which describes the case of a woman brought to an emergency department by the police after threatening to jump off a bridge. The woman receives psychosocial support from a nurse and her suicidal ideation resolves. Psychosocial interventions are portrayed as highly effective. Unfortunately, there is very little discussion of solutions that may need to be used should such interventions prove ineffective. It is well recognised that a collaborative multidisciplinary approach provides the best possible model of care for patients. Psychosocial and supportive interventions are often exhaustively employed prior to the use of coercive measures.

\section{Portrayal of involuntary practices}

Within the QualityRights materials there are numerous times where gross human rights violations are presented alongside involuntary treatments, in a way that implies that these practices are equivalent. Involuntary interventions remain legal in the majority of jurisdictions and until recently were endorsed by the WHO.

For example, the Human Rights module describes a woman who is given medication in hospital that made her feel unwell, and she is also subjected to violence and abuse while an in-patient. The material is written in a manner that suggests these two events are equivalent. The module on mental health, disability and human rights includes the sentence 'they [people with disabilities] should not be tortured, beaten, raped or otherwise abused, they should not be given treatment or surgery without their consent, they should not be sterilized against their will'. Psychiatrists may find it disturbing to know that the WHO views elements of their practice as torture.

\section{Acceptance of the criminalisation of individuals with severe mental illness}

There has been much debate around Article 12 of the CRPD and the potential for it to either protect or undermine the rights of people with mental disabilities. Article 12 provides for 'equal recognition before the law'. Freeman et al have argued that granting all people legal capacity at all times irrespective of mental status actually undermines critical rights of people with mental health disability and can jeopardise people's quality of life, their health and their right to liberty. ${ }^{2}$

Careful consideration must be given to the alternatives to coercive practices and the very real danger of criminalising people who are mentally unwell must be born in mind. The QualityRights Initiative appears to be disturbingly accepting of this. ${ }^{5}$ The 'Legal capacity and the right to decide' module, states that 'People with disabilities can only be detained on the same basis (or for the same reasons) as all other citizens (e.g. following a criminal sentence)' (p. 29). ${ }^{1}$ In the QualityRights module on seclusion and restraint it is suggested an individual who poses a risk to themselves or others 'should be stopped in the same manner as you would stop anyone, with or without a disability - such as by involving a specially trained group who are equipped with the skills to manage the situation (e.g. a response team, see topic 10) or alternatively in some instances law enforcement bodies (e.g. the police force)' (p. 21). ${ }^{1}$

Sadly, the role of law enforcement managing individuals with acute episodes of mental illness has resulted in many high-profile deaths and use of excessive force. The use of coercive measures by mental health staff is far from ideal, but the alternative suggestions are more problematic.

\section{Conclusions}

Despite the concerns raised above, the QualityRights Initiative should have a central role in helping realise the CRPD, limiting restrictive practices and promoting individual autonomy. Many of the suggested practices and initiatives described in the training material are easily implementable and sadly long overdue. However, the negative portrayal of psychiatry and medication in the training material is unhelpful. The vast majority of psychiatrists approach coercive practices as a last resort, after a careful weighing of the risks and benefits and often after extensive discussion with the individual receiving treatment and their family members. Although there is a clear need for enhancing the use of psychosocial interventions and multidisciplinary contribution, these practices are not universally effective. Characterising involuntary treatments as human rights abuses unnecessarily polarises an important debate. Failure to consider contingency plans for individuals with severe mental illness may lead to treatable conditions remaining untreated or to mental illness being addressed in a forensic setting. The QualityRights Initiative appear worryingly accepting of this.

Fiona Hoare (D), Department of Psychiatry, The Mater Misericordiae University Hospital, Ireland; Richard M. Duffy, Department of Psychiatry, The Mater Misericordiae University Hospital, Ireland

Correspondence: Fiona Hoare. Email: fiona.hoare@ucdconnect.ie

First received 21 Nov 2020, final revision 6 Jan 2021, accepted 30 Jan 2021

\section{Author contribution}

Both authors reviewed the WHO QualityRights Initiative and training materials for the purposes of this paper. They were both involved in the drafting of the editorial and subsequent revisions. Both authors had final approval of the version submitted for publication and agree to be accountable for all aspects of the work. 


\section{Declaration of interest}

Richard Duffy is a member of the Human Rights and Ethics Committee in the College of Psychiatrists of Ireland.

\section{References}

1 World Health Organization. QualityRights Materials for Training, Guidance and Transformation. WHO, 2019 (https://www.who.int/publications/i/item/whoqualityrights-guidance-and-training-tools).
2 Freeman MC, Kolappa K, de Almeida JMC, Kleinman A, Makhashvili N, Phakathi $\mathrm{S}$, et al. Reversing hard won victories in the name of human rights: a critique of the general comment on article 12 of the UN convention on the rights of persons with disabilities. Lancet Psychiatry 2015; 2: 844-50.

3 Funk M, Drew N. WHO QualityRights: transforming mental health services. Lancet Psychiatry 2017; 4: 826-7.

4 Pathare S, Funk M, Drew Bold N, Chauhan A, Kalha J, Krishnamoorthy S, et al. Systematic evaluation of the QualityRights programme in public mental health facilities in Gujarat, India. Br J Psychiatry [Epub ahead of print] 20 June 2019. Available from: https://doi.org/10.1192/bjp.2019.138.

5 Duffy RM, Kelly BD. World Health Organization and Mental Health Law. In India's Mental Healthcare Act, 2017: Building Laws, Protecting Rights (eds RM Duffy, BD Kelly): 25-48. Springer, 2020. psychiatry in history

\section{Epidemics: wash your hands! The asylum delivery and violent death of Professor Ignaz Philipp Semmelweis; and, the cursed Semmelweis reflex}

\author{
Greg Wilkinson (iD
}

That hand hygiene is the single most important factor in the control of infection flows from Semmelweis (1818-1865), a Hungarian assistant obstetrician, who deduced in 1847 that the incidence of puerperal fever in Vienna's Allgemeines Krankenhaus could be minimised by the use of hand disinfection in a chlorinated lime solution. Alas, Semmelweis did not live to reap the full fruits of his discovery, which was rejected, ridiculed and not widely adopted until after his death, via Pasteur's confirmation of the germ theory of disease (1860s) and Lister's (1865) successful surgical practice using a carbolic acid solution.

Codell Carter et al (1995) relate from five surviving documents that on 13 July 1865, now Professor of Obstetrics at Pest, Semmelweis's behaviour was so inappropriate that his wife thought he might be losing his mind. Two weeks later he was examined by his friend, a paediatrician, Dr Bókai, who concluded that he required specialist attention. For 5 weeks his personal, sexual, professional and social behaviour had changed: he drank alcohol immoderately, went with a prostitute, wasted money, his nights were restless, his appetite great, he sweated profusely and he drank water excessively: affective or infective?

On 29 July 1865, Jánoš Balassa, Professor of Surgery, with Dr Bókai and Professor Wagner, a physician, wrote a referral committing Semmelweis to a Viennese asylum. That evening, Semmelweis travelled with family to Vienna by overnight train on his way, he thought, to a German spa but, on arrival in Vienna next morning, on a pretext a former colleague, Ferdinand Hebra, a dermatologist, delivered him to the public asylum in Lazarettgasse near the Krankenhaus. There is no evidence that Semmelweis was examined or interviewed on admission, of who was in charge of him and who compiled the inconsistent records, contemporaneously or post hoc.

Initially he was excited, restless, confused, grandiose and behaving bizarrely: hot head, pulse 120 (subsequently 140). On the middle finger of the right hand there was contusion or gangrene, which he reportedly said 'appeared of itself' (but was not described pre-admission). He was intermittently put in a straitjacket. He fought with the attendant because he would not let him out, and he wanted to jump out of the window. Three (some say six) attendants could hardly control him. Ultimately, he was reportedly blue in the face, trembling and breathing with difficulty: gangrene worsened, boils were everywhere on his extremities and he had 'An abscess (?) corresponding to the left thorax'.

Semmelweis died on 13 August 1865, aged 47 . His body was dissected where he had performed autopsies seeking the cause of puerperal fever. His cause of death was, ironically, infection - pyaemia: acute gangrenous osteomyelitis of the middle finger of the right hand and a metastatic abscess extending into the thorax, with 'in the left thorax an ichor source the size of a man's fist' - the said consequence of untreated injuries that Semmelweis received in the asylum.

The Semmelweis reflex is the reflex-like rejection of new knowledge because it contradicts entrenched norms, beliefs or paradigms.

(c) The Author(s), 2021. Published by Cambridge University Press on behalf of the Royal College of Psychiatrists 